RESEARCH ARTICLE

\title{
Coherent optical vortex generation with multiple topological charges based on a seeded free electron laser
}

\author{
Hao Sun ${ }^{1,2}$, Xiaofan Wang ${ }^{3,4}$, Chao Feng ${ }^{5}$, Lingjun Tu ${ }^{1,2}$, Weijie Fan ${ }^{1,2}$, and Bo Liu ${ }^{5}$ \\ ${ }^{1}$ Shanghai Institute of Applied Physics, Chinese Academy of Sciences, Shanghai 201800, China \\ ${ }^{2}$ University of Chinese Academy of Sciences, Beijing 100049, China \\ ${ }^{3}$ Institute of Advanced Science Facilities, Shenzhen 518000, China \\ ${ }^{4}$ Southern University of Science and Technology, College of Science, Shenzhen 518055, China \\ ${ }^{5}$ Shanghai Advanced Research Institute, Chinese Academy of Sciences, Shanghai 201210, China \\ (Received 1 August 2021; revised 15 September 2021; accepted 19 November 2021)
}

\begin{abstract}
To generate optical vortex with multiple topological charges, a simple scheme based on the phase mask shaping technique is proposed and applied in a seeded free electron laser. With a tailored phase mask, an extreme-ultraviolet (EUV) vortex with multiple topological charges can be produced. To prove the feasibility of this method, an eight-step phase mask is designed to shape the seed laser. The simulation results demonstrate that 100-MW, fully coherent EUV vortex pulses with topological charge 2 can be generated based on the proposed technique. We have also demonstrated the possibility of generating higher topological charges by using a phase mask with more steps.
\end{abstract}

Keywords: free electron laser; high-gain harmonic generation; optical vortex; phase mask

\section{Introduction}

Starting from the work of Allen et al..$^{[1]}$, the optical vortices which carry orbital angular momentum (OAM), or light beams with spatial phase dependence of $\exp (i \ell \phi)$, where $\phi$ denotes the azimuthal coordinate and $l$ denotes an integer referring to the topological charge, have become the subject of intense interest for numerous applications, including micromanipulation ${ }^{[2]}$, detection of spinning objects ${ }^{[3]}$, microscopy ${ }^{[4]}$, imaging ${ }^{[5]}$, optical pump schemes $^{[6]}$, and communication ${ }^{[7]}$. In the extreme-ultraviolet (EUV) wavelength range, the interaction of the vortex light with matter can produce special physical phenomena. Among these are the formation of Skyrmionic defects ${ }^{[8]}$, the formation of charge current loops in fullerenes with an associated orbital magnetic moment ${ }^{[9]}$ and the violation of dipolar selection principles during photoionization ${ }^{[10]}$. In the visible and infrared-wavelength regimes, the optical vortex

Correspondence to: $\mathrm{X}$. Wang, Institute of Advanced Science Facilities, Shenzhen 518000, China; C. Feng and B. Liu, Shanghai Advanced Research Institute, Chinese Academy of Sciences, Shanghai 201210, China. Email: wangxf@mail.iasf.ac.cn (X. Wang); fengchao@zjlab.org.cn (C. Feng); liubo@zjlab.org.cn (B. Liu) could be generated by a spiral phase plate ${ }^{[11]}$, the cylindrical mode converter ${ }^{[12]}$, computer-generated holograms ${ }^{[13]}$, and so on. At shorter wavelengths, such as in the EUV and X-ray regime, these techniques are no longer applicable because of the strong radiation damage of optical elements.

To generate short-wavelength vortex beams, some schemes based on free electron lasers (FELs) have been proposed. As exceptionally bright, coherent photon sources for short-wavelength radiation, FELs ${ }^{[14]}$ provide scientists with formidable instruments for exploring the structural characteristics of materials at the atomic scale. However, the transverse profiles of radiation from the undulator are limited to a fundamental Gaussian-like mode with no azimuthal phase variation. For the creation of vortex beams, one method based on helical undulator harmonic radiation was proposed ${ }^{[15]}$. The method is based on selfamplified spontaneous emission $(\mathrm{SASE})^{[16]}$ principle, which suffers from the limited temporal coherence as the amplification starts from random noise in the e-beam longitudinal distribution. To achieve fully temporally coherent radiation ${ }^{[17]}$, seeded FELs schemes relying on e-beam phase space manipulation on the optical wavelength scale, such as high-gain harmonic generation $(\mathrm{HGHG})^{[18]}$ and 
echo-enabled harmonic generation $(\mathrm{EEHG})^{[19]}$, have been proposed and experimentally demonstrated ${ }^{[20,21]}$. To produce coherent optical vortex, these seeded FEL schemes have been modified. For the HGHG scheme, a Gaussian seed laser is superimposed on the electron beam in a helical undulator $^{[22]}$. The helically bunched beam can produce optical vortex at the fundamental frequency of the seed in the radiator. This method can be used as a mode converter which requires a coherent EUV or X-ray input signal. Ribič and his coworkers modified the HGHG scheme, which used a four staircase-like-phase mask to modify the seed laser ${ }^{[23]}$. However, the method can only generate FEL vortex light with topological charge 1. For some special pump-probe experiments $^{[6]}$, vortex beams with multiple topological charges are needed. For the EEHG scheme, Hemsing and his collaborators proposed the echo-enabled X-ray vortexgeneration scheme where at least one vortex seed laser is used to produce corkscrew microbunching ${ }^{[24]}$. However, this scheme is more complex and has not been proved experimentally.

To produce vortex beams with multiple topological charges, we propose a simple method based on using the phase mask shaping technique. The topological charge of vortex light can be controlled by tailoring the structure of the phase mask. In this paper, to prove the feasibility of this method, an eight-step phase mask is designed to shape the seed laser. The simulation result demonstrates that 100-MW, fully coherent EUV vortex pulses with topological charge 2 can be generated based on this phase mask shaping technique. We also show the possibility of generating vortex light with higher topological charges by using a phase mask with more steps. The vortex generation scheme based on HGHG can be straightforwardly implemented at the existing seeded FEL user facilities such as Shanghai Soft X-Ray Free Electron Laser (SXFEL) ${ }^{[25]}$ and FERMI ${ }^{[21]}$ without the need for significant machine upgrades.

The remainder of this paper is organized as follows. In Section 2, the principle of the proposed scheme is illustrated. In Section 3, the optical design of this scheme is shown. In Section 4, the interaction between the electron beam and the optical field is investigated by theory and simulation. In Section 5, we study the FEL performance in the radiator. In Section 6, we present some results and considerations for generating the vortex light with higher topological charges. Finally, we give a discussion in Section 7.

\section{Principle}

The schematic layout of the proposed technique is shown in Figure 1. This scheme is based on the HGHG setup. First, the electron beam interacts with the seed laser in the modulator. The seed properties such as the transverse phase dependence are imprinted onto the electron beam. When the electron beam passes through the chicane, the energy modulation is converted into a coherent density modulation rich in harmonic content. The second undulator is tuned to a higher harmonic of the seed laser, causing the electron beam to emit coherent radiation at a shorter wavelength.

The relative transverse phase changes of the seed laser after passing through the phase mask as shown in Figure 1 can be represented as a matrix:

$$
\text { Phase }_{\text {mask }}=\left[\begin{array}{cccc}
\frac{3 \pi}{2} & \pi & \frac{\pi}{2} & 0 \\
0 & \frac{\pi}{2} & \pi & \frac{3 \pi}{2}
\end{array}\right] .
$$

Each number in this matrix represents the phase value at each interval $\frac{\pi}{4}$ radians azimuth position. In a short modulator, the electron beam interacts with the seed laser which travels through the phase mask. The transverse eight-step phase structure is imprinted onto the electron beam. The electron beam phase structure can also be represented as shown in Equation (1).

After the electron beam passes through the dispersion section, the above energy modulation is converted into a coherent microbunching. The transverse phase of microbunching can be represented as in the matrix in Equation (1). According to the frequency up-conversion theory of $\mathrm{HGHG}^{[18]}$, the transverse phase structure of the electron microbunching at the radiator entrance can be interpreted as the following matrix:

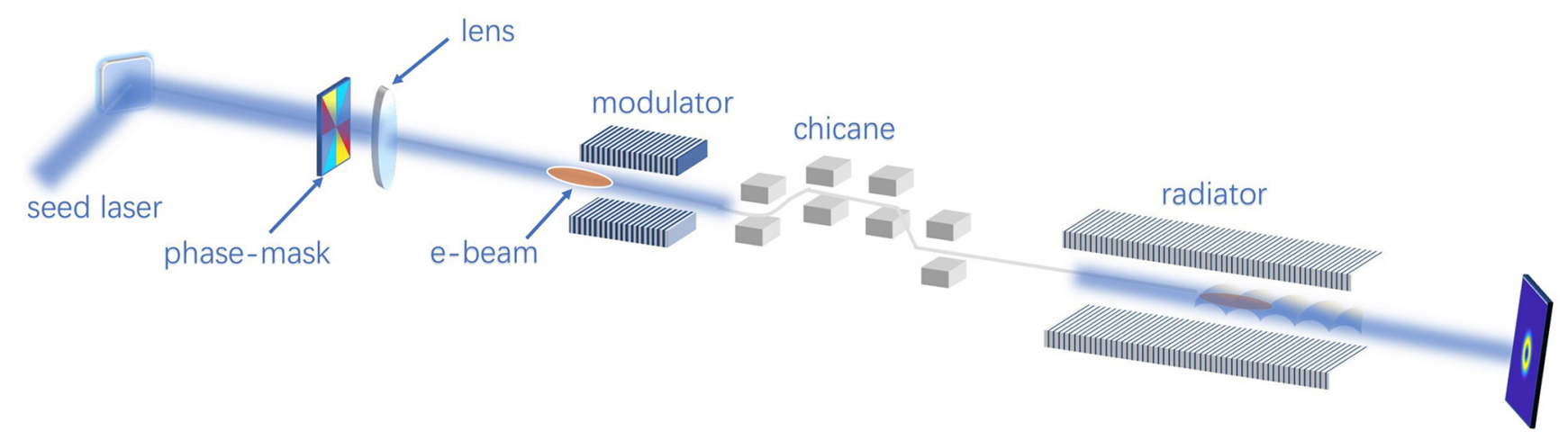

Figure 1. Scheme to generate an optical vortex with multiple topological charges based on an HGHG setup. 


$$
\text { Phase }_{\text {rad }}=n \cdot \text { Phase }_{\text {ebeam }}=n \cdot\left[\begin{array}{cccc}
\frac{3 \pi}{2} & \pi & \frac{\pi}{2} & 0 \\
0 & \frac{\pi}{2} & \pi & \frac{3 \pi}{2}
\end{array}\right] \bmod 2 \pi \text {, }
$$

where $n$ is the harmonic number. The final electron microbunching structure will be different for different values of $n$. For the case where $n$ is an even number, the electron microbunching structure can be represented as

$$
\text { Phase }_{\mathrm{rad}}=\left[\begin{array}{llll}
0 & 0 & 0 & 0 \\
0 & 0 & 0 & 0
\end{array}\right](n=4 m),
$$

and

$$
\text { Phase }_{\mathrm{rad}}=\left[\begin{array}{llll}
\pi & 0 & \pi & 0 \\
\pi & 0 & \pi & 0
\end{array}\right](n=4 m+2) .
$$

It should be noted that after up-conversion, the phase jump of each step in the microbunching is 0 or $\pi$ when $n$ is an even number. However, this phase structure is not suitable for generating an optical vortex. When $n$ is an odd number $n=2 m+1$, the electron microbunching structure can be represented as

$$
\text { Phase }_{\mathrm{rad}}=\left[\begin{array}{cccc}
\frac{3 \pi}{2} & \pi & \frac{\pi}{2} & 0 \\
0 & \frac{\pi}{2} & \pi & \frac{3 \pi}{2}
\end{array}\right](m: \text { even }),
$$

and

$$
\text { Phase }_{\mathrm{rad}}=\left[\begin{array}{cccc}
\frac{\pi}{2} & \pi & \frac{3 \pi}{2} & 0 \\
0 & \frac{3 \pi}{2} & \pi & \frac{\pi}{2}
\end{array}\right](m: \text { odd })
$$

For the case of odd $n$, it can be shown that the final transverse phase of e-beam increases in the opposite direction for different values of $m$. According to the above theory, the electron beam will emit light with an eight-step structure in the radiator entrance at a high odd harmonic of the seed laser. According to the analysis, the eight-step phase mask can be created for seed laser shaping and, hence, for fine transverse shaping of the electron beam in the modulator. In general, the phase of the microbunching is constant in the transverse plane. In this scheme, the transverse phase of the microbunching is an eight-step structure which has a helical bunching component at high harmonic frequency.
Therefore, the radiation emitted by the special electron beam will develop into the optical vortex along the radiator.

\section{The principle of phase mask shaping}

The optical setup for the seed laser in the proposed scheme is shown in Figure 2, where the seed laser is focused through a lens immediately after passing through the phase mask and finally injected into the undulator to interact with the electron beam.

The transport processes of the seed laser can be studied with the wave optics ${ }^{[26]}$. The optical field after the phase mask is $\tilde{E}\left(x_{1}, y_{1}\right)$. The complex optical field after lens is given by the following formula

$$
\tilde{E}^{\prime}\left(x_{1}, y_{1}\right)=\tilde{E}\left(x_{1}, y_{1}\right) \exp \left[-\frac{i k}{2 f}\left(x_{1}^{2}+y_{1}^{2}\right)\right] \text {. }
$$

Here $f$ is the focal length of the lens and $k$ denotes the wave number of the laser. According to the Fresnel optical propagation theory, the light field after a distance $f$ is

$$
\begin{aligned}
E(x, y)= & \frac{\exp (i k f)}{i \lambda f} \exp \left[\frac{i k}{2 f}\left(x^{2}+y^{2}\right)\right] \\
& \times \mathcal{F}\left\{\tilde{E}^{\prime}\left(x_{1}, y_{1}\right) \exp \left[\frac{i k}{2 f}\left(x_{1}^{2}+y_{1}^{2}\right)\right]\right\} .
\end{aligned}
$$

Equation (8) could be mathematically simplified to

$$
E(x, y)=\frac{\exp (i k f)}{i \lambda f} \exp \left[\frac{i k}{2 f}\left(x^{2}+y^{2}\right)\right] \mathcal{F}\left\{\tilde{E}\left(x_{1}, y_{1}\right)\right\}
$$

The above formula shows that the complex optical field in the focal plane is the Fourier transform of the optical field through phase mask and the complex optical field can remain the eight-step structure in the focal plane. The above equations are written into a program to model and analyze the optical field propagation process. The complex optical field in the focal plane is shown in Figure 3. The transverse phase of the optical field presents a distinct eightstep structure.

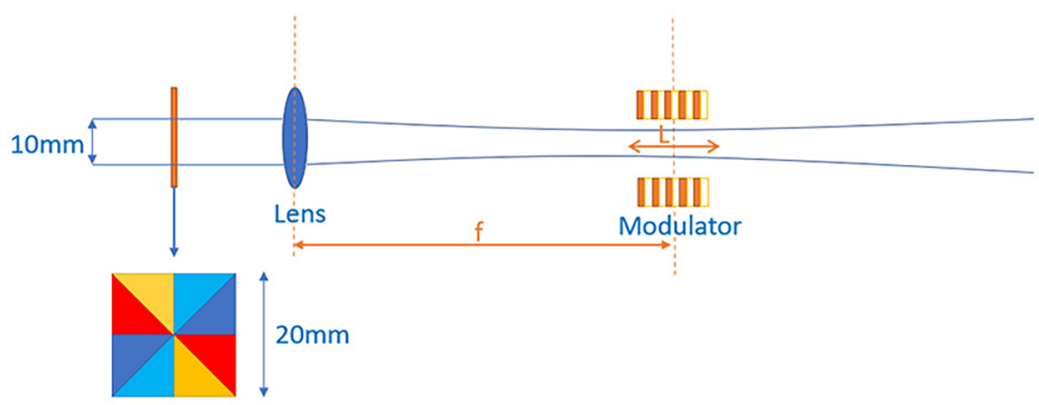

Eight-step phase mask

Figure 2. Optical setup in this scheme. 


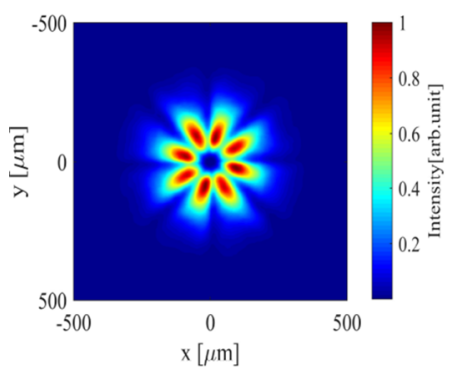

(a)

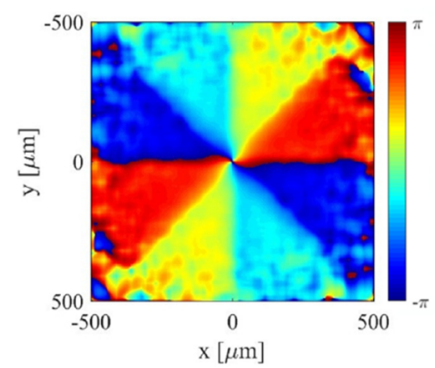

(b)

Figure 3. (a) Transverse intensity and (b) corresponding phase of seed laser in the focal plane.

In the proposed scheme, the focal plane of the lens is set to the middle of the modulator as shown in Figure 2. According to the propagation theory of wave optics, the optical field distribution after transverse shaping to a certain place is related to the focal length of the previous lens. In addition, the lens can suppress the divergence of the light field near the focal plane. Here, a lens with long focal length exceeding $20 \mathrm{~m}$ and a short modulator with length of $50 \mathrm{~cm}$ are chosen to maintain the eight-step structure of the laser beam in the modulator.

\section{Interaction between the electron beam and the optical field}

A three-dimensional algorithm ${ }^{[27,28]}$, which is built based on the fundamental basis of electrodynamics, is modified for simulating the laser-beam interaction in the modulator. The magnetic field distribution of the planar undulator in the $y$ direction can be written as

$$
B_{y}=B_{0} \sin \left(k_{\mathrm{u}} z\right),
$$

where $B_{0}$ is the undulator peak magnetic field, $k_{\mathrm{u}}=2 \pi / \lambda_{\mathrm{u}}$ is the wave number of the undulator, and $\lambda_{\mathrm{u}}$ is the period length of the undulator. The results in Section 3 demonstrate that the transverse phase of the complex field near the focal plane can be maintained in an eight-step structure. Thus, the electric field of a seed laser near the focal plane can be represented as

$$
E_{x}=E_{0} \sin \left[k_{\mathrm{s}}\left(z^{\prime}-z_{0}\right)+\phi\right] \sqrt{e^{-\frac{x^{2}}{2 \sigma_{x}^{2}}-\frac{y^{2}}{2 \sigma_{y}^{2}} \frac{\left(z^{\prime}-z_{0}\right)^{2}}{2 \sigma_{z}^{2}}}},
$$

and

$$
\phi=\left[\begin{array}{cccc}
\frac{3 \pi}{2} & \pi & \frac{\pi}{2} & 0 \\
0 & \frac{\pi}{2} & \pi & \frac{3 \pi}{2}
\end{array}\right]
$$

where $k_{\mathrm{s}}=2 \pi / \lambda_{\mathrm{s}}$ is the wave number of the seed laser, $z_{0}$ is the initial relative position of the laser from the electron beam, $E_{0}$ is the peak electric field intensity of the seed laser, and $\phi$ is the carrier envelope phase of the seed laser. For the optical field with eight-step transverse distribution, the
Table 1. Main parameters of the simulation.

\begin{tabular}{ll}
\hline Parameter & Value \\
\hline Beam energy & $1.5 \mathrm{GeV}$ \\
Peak current & $1200 \mathrm{~A}$ \\
Emittance & $1 \mathrm{~mm} \cdot \mathrm{mrad}$ \\
Energy spread (rms) & $150 \mathrm{keV}$ \\
Seed wavelength & $266 \mathrm{~nm}$ \\
Seed power & $2000 \mathrm{MW}$ \\
Modulator length & $0.5 \mathrm{~m}$ \\
Radiator period & $0.05 \mathrm{~m}$ \\
Radiation wavelength & $38 \mathrm{~nm}$ \\
\hline
\end{tabular}

phase can be represented as the above matrix similar to the transverse phase of the phase mask referred to Section 2. The diffraction effects of the laser field can be expressed by

$$
\sigma_{x}(z)=\sqrt{\sigma_{x \mathrm{w}}^{2}+\frac{k_{\mathrm{s}}^{2}\left(z-z_{\mathrm{w}}\right)^{2}}{4 \sigma_{x \mathrm{w}}^{2}}},
$$

and

$$
\sigma_{y}(z)=\sqrt{\sigma_{y \mathrm{w}}^{2}+\frac{k_{\mathrm{s}}^{2}\left(z-z_{\mathrm{w}}\right)^{2}}{4 \sigma_{y \mathrm{~W}}^{2}}},
$$

where $\sigma_{x \mathrm{w}}$ and $\sigma_{y \mathrm{w}}$ denote the laser size at the longitudinal waist position $z_{\mathrm{w}}$. In the undulator, the electron's motion satisfies the law of the electrodynamics,

$$
\gamma m \frac{\mathrm{d} \vec{v}}{\mathrm{~d} t}=e \vec{E}-e \vec{v} \times \vec{B}
$$

The energy exchange between the electrons and the laser field in the undulator can be represented as

$$
m c^{2} \frac{\mathrm{d} \gamma}{\mathrm{d} t}=e E_{x} \frac{\mathrm{d} x}{\mathrm{~d} t}
$$

Parameters of the Shanghai Soft X-Ray Free Electron Laser User Facility (SXFEL-UF) are used here for the simulation, as summarized in Table 1. The seed laser originates from a commercial Ti:sapphire laser system, which can provide 


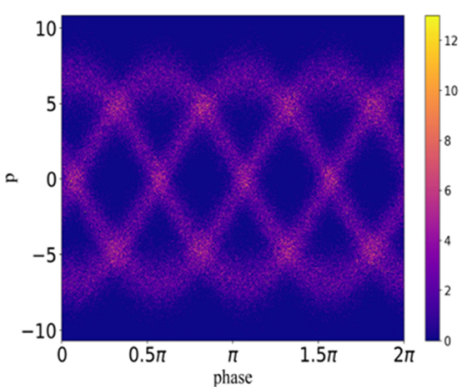

(a)

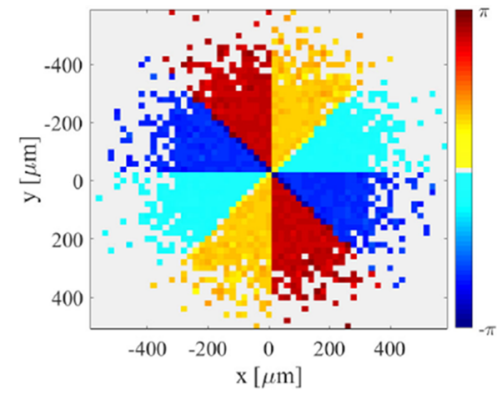

(b)

Figure 4. (a) Longitudinal phase space of the electron beam after the modulator. The vertical axis $p$ represents the energy modulation amplitude, which is defined as the ratio of the electron's deviation from the central energy to the initial energy spread. (b) Transverse phase of the local microbunching $b(\vec{r})$.

laser pulses with pulse energy up to $10 \mathrm{~mJ}$ at $800 \mathrm{~nm}$. A third harmonic generation (THG) is employed to convert the laser to $266 \mathrm{~nm}$. Even with a conversion efficiency of $10 \%$ of the THG, it is easy to achieve a peak power of $10 \mathrm{GW}$ level at $266 \mathrm{~nm}$. Here we adopted a seed laser power of $2 \mathrm{GW}$ in the simulations. With these parameters, the electron beam receives about seven times the energy modulation of its energy spread. The longitudinal phase space after the modulator is shown in Figure 4(a).

Unlike the common sinusoidal energy modulation of an electron beam, there are several discrete sinusoidal modulations in an optical wavelength with phase delays between them. As shown in Figure 1, the electron beam interacts with the seed laser field which has typical transverse phase structure in the modulator. The seed properties such as the transverse phase dependence are imprinted on the electron beam phase space. Then the electron beam passes through the dispersion section for density modulation where different energy electrons travel different lengths. As a result of this process, the microbunching structure in electron beam is created. Considering the transverse structure of the microbunching, the local bunching factor can be calculated as $^{[23]}$

$$
b(\vec{r})=\left\langle\exp \left[i \theta_{i}(\vec{r})\right]\right\rangle
$$

Here, $\theta_{i}$ is the pondermotive phase of the $i$ th particle and the brackets represent the ensemble average over all the particles at a given transverse position $\vec{r}$ in the electron beam. Figure 4(b) illustrates the transverse phase of the local microbunching factor $b(\vec{r})$, where the transverse phase of the microbunching is not constant but has an eight-step structure.

\section{FEL performance}

The FEL radiation is simulated with the three-dimensional time-dependent FEL simulation code Genesis $1.3^{[29]}$. The radiator is resonant at the seventh harmonic of the seed laser, which is $38 \mathrm{~nm}$. The microbunched electron beam as shown in Figure 4(b) is sent into the radiator with proper lattice arrangement for FEL simulation. As illustrated in Figure 5(a), the coherent radiation produced by the modulated electron beam retains the eight-step structure at the radiator entrance. Consequently, the evolution of the radiation profile is primarily governed by the diffraction and linear field amplification. Then, the radiation profile gradually evolves from an eight-step structure to a ring-like distribution with helical transverse phase. At the radiator exit, the radiation profile changes to a typical vortex light profile with topological charge 2 and the radiation power is saturated. To illustrate the evolution of electron beam in the radiator, the helical bunching factor has been illustrated in Figure 5(b) according to the approaches mentioned in the paper ${ }^{[24]}$. The electron bunching factor at the azimuthal mode $l$ is denoted by

$$
b_{l}(k)=\left|\left\langle e^{-i k z^{\prime \prime}-i l \phi} f\left(\mathbf{x}_{\perp}, z, p\right)\right\rangle\right|
$$

where $f\left(\mathbf{x}_{\perp}, z, p\right)$ is the electron beam distribution and brackets indicate averaging over the ultimate coordinates, and $\mathbf{x}_{\perp}=(r, \phi)$ represents the radial and azimuthal coordinates. Here, $k$ is the harmonic frequency, $z$ is the longitudinal position of the electron beam, and $p$ is the energy modulation amplitude:

$$
p=\left(E-E_{0}\right) / \sigma_{\mathrm{E}}
$$

where $E_{0}$ is the central beam energy and $\sigma_{\mathrm{E}}$ is the rms energy spread. As shown in Figure 5(b), $b_{2}$ is always dominant in the radiator and $b_{0}$ and $b_{1}$ are suppressed. Consequently, this allows the optical vortex with topological charge 2 to dominate the radiation in the mode competition along the radiator. The radiation with topological charges 1 and 0 is suppressed in the radiator. Figures 5(c) and 5(d) show the longitudinal profile of the radiation pulse and the corresponding spectrum at the radiator exit. The simulation results show that 100-MW, fully coherent EUV vortex pulses with topological charge 2 at $38 \mathrm{~nm}$ can be generated at the exit of radiator. Figure 6 shows the final transverse intensity and its 


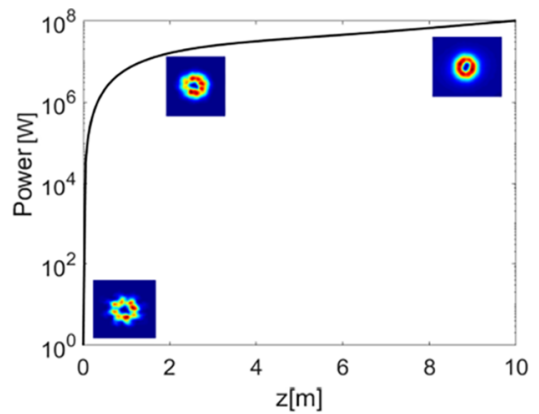

(a)

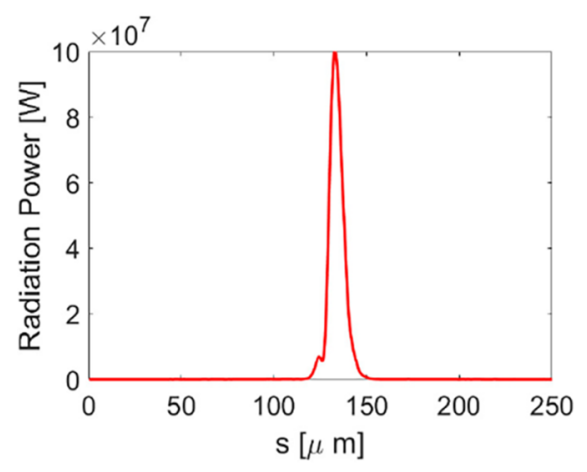

(c)

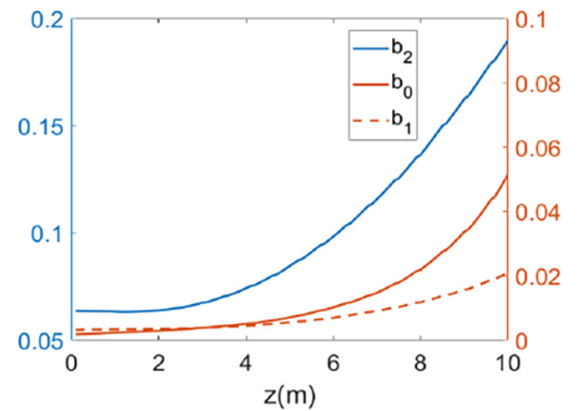

(b)

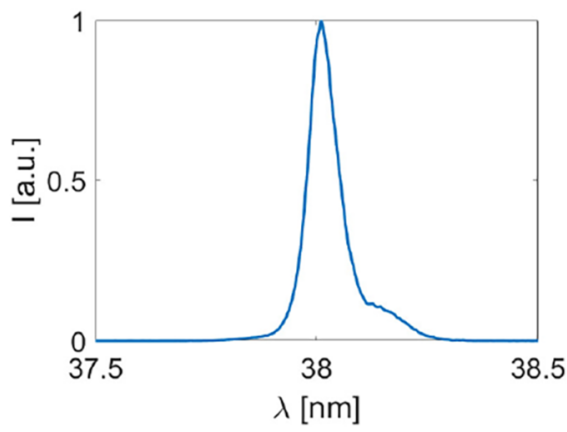

(d)

Figure 5. (a) Evolution of the power along the radiator. (b) Evolution of the bunching factor along the radiator. (c) The longitudinal power and (d) its spectrum at the radiator exit.

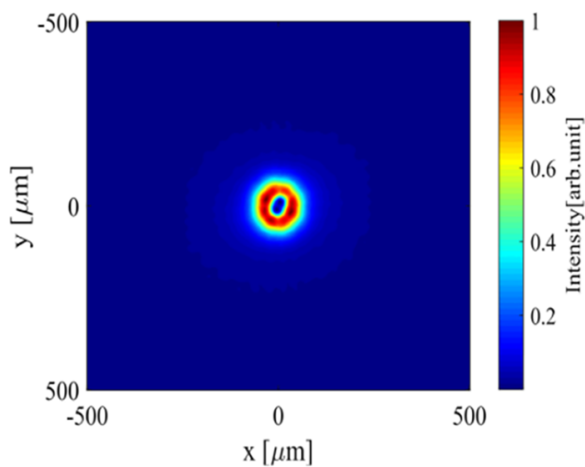

(a)

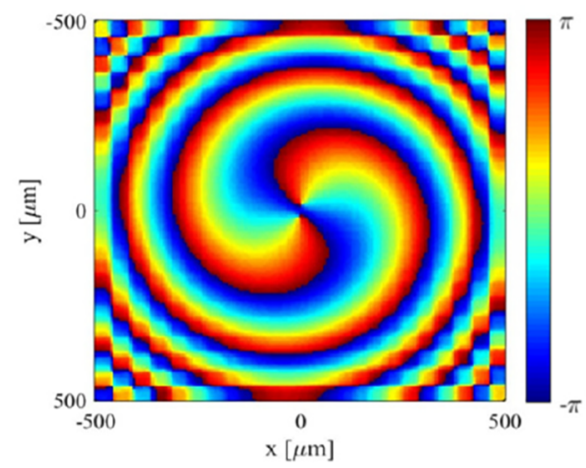

(b)

Figure 6. (a) The transverse intensity and (b) its corresponding phase of radiation at the radiator exit.

corresponding phase of radiation at the radiator exit. It can be clearly seen that the transverse intensity distribution of the light presents an obvious ring-like profile, and the transverse phase distribution has the typical characteristics of vortex light with topological charge 2 .

\section{Generation of vortex light with higher topological charges}

The phase mask shaping technique is also suitable for the generation of vortex light with higher topological charges.
Here we take a phase plate with 12 steps as an example to show the generation of vortex beam with topological charge 3 at $38 \mathrm{~nm}$. Simulation results are given in Figure 7. By using an undulator segment with length of $4 \mathrm{~m}$, vortex radiation pulse with peak power over $10 \mathrm{MW}$ can be generated. The transverse phase distribution has the typical characteristics of vortex light with topological charge 3 , as shown in Figure 7(b). However, it should be noted that the vortex light with topological charge 3 can hardly be amplified to saturation due to the weaker transverse coupling between the electron beam and the vortex light with greater emission angle ${ }^{[24]}$. 


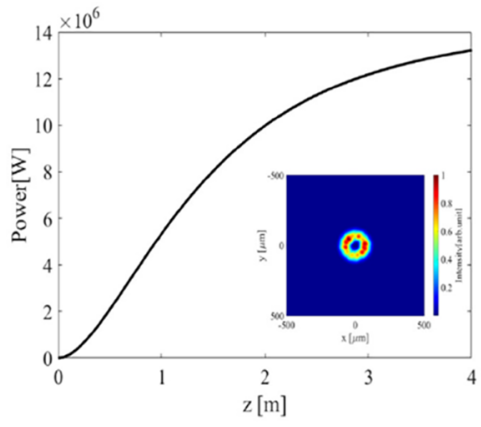

(a)

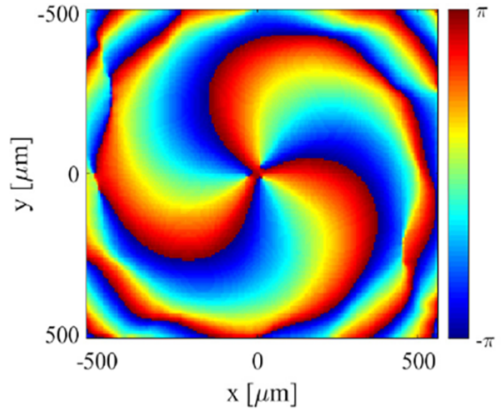

(b)

Figure 7. (a) Evolution of the power along the radiator. (b) The transverse phase of radiation at the radiator exit.

\section{Discussion and conclusion}

A novel technique based on phase mask shaping has been proposed in this paper to generate optical vortex with multiple topological charges in seeded FELs. Through a fine transverse manipulation of the seed laser, a multidimensional modulation of the electron beam can be achieved to generate the optical vortex with multiple topological charges. Therefore, the spatial-temporal properties of the radiation generated in an undulator can be manipulated. In this paper, to produce optical vortex with multiple topological charges, an eight-step phase mask scheme has been devised for the transverse shaping of the seed laser. The final transverse shaping of microbunching can also be changed due to the HGHG principle. The simulation results show that $100-\mathrm{MW}$, fully coherent EUV vortex pulses with topological charge 2 can be generated based on the proposed scheme. The topological charge of vortex light can be controlled by tailoring the structure of the phase mask. The simulation results also show the EUV vortex with topological charge 3 can be produced with sufficient power. These results show that the proposed scheme is feasible to generate EUV vortex light with multiple topological charges. We found in the simulations that the generated vortex light with topological charges higher than 2 cannot be effectively amplified to saturation due to the weaker transverse coupling between the electron beam and the vortex light with greater emission angle in the undulator. Further studies on this topic are still ongoing. The principle-proving experiments of generating optical vortex based on four stairs and eight-step phase mask are under preparation at the SXFEL.

\section{Acknowledgments}

The authors would like to thank Primoz Rebernik Ribič and Yujie Lu for fruitful discussions in free lectron laser physics and simulations. This work was supported by the National Natural Science Foundation of China (Nos. 12122514 and
11975300) and Shanghai Science and Technology Committee Rising-Star Program (No. 20QA1410100).

\section{References}

1. L. Allen, M. W. Beijersbergen, R. Spreeuw, and J. Woerdman, Phys. Rev. A 45, 8185 (1992).

2. H. He, M. Friese, N. Heckenberg, and H. Rubinsztein-Dunlop, Phys. Rev. Lett. 75, 826 (1995).

3. M. P. Lavery, F. C. Speirits, S. M. Barnett, and M. J. Padgett, Science 341, 537 (2013).

4. A. Jesacher, S. Fürhapter, S. Bernet, and M. Ritsch-Marte, Phys. Rev. Lett. 94, 233902 (2005).

5. K. Liu, Y. Cheng, Y. Gao, X. Li, Y. Qin, and H. Wang, Appl. Phys. Lett. 110, 164102 (2017).

6. K. Shigematsu, K. Yamane, R. Morita, and Y. Toda, Phys. Rev. B 93, 045205 (2016).

7. M. Krenn, R. Fickler, M. Fink, J. Handsteiner, M. Malik, T. Scheidl, R. Ursin, and A. Zeilinger, New J. Phys. 16, 1088 (2014).

8. H. Fujita and M. Sato, Phys. Rev. B 95, 054421 (2017).

9. J. Wätzel, Y. Pavlyukh, A. Schäffer, and J. Berakdar, Carbon 99, 439 (2016).

10. A. Picón, A. Benseny, J. Mompart, J. V. de Aldana, L. Plaja, G. F. Calvo, and L. Roso, New J. Phys. 12, 083053 (2010).

11. V. V. Kotlyar, A. A. Almazov, S. N. Khonina, V. A. Soifer, H. Elfstrom, and J. Turunen, J. Opt. Soc. Am. A 22, 849 (2005).

12. M. W. Beijersbergen, L. Allen, H. van der Veen, and J. Woerdman, Opt. Commun. 96, 123 (1993).

13. B. Terhalle, A. Langner, B. Päivänranta, V. A. Guzenko, C. David, and Y. Ekinci, Opt. Lett. 36, 4143 (2011).

14. P. Emma, R. Akre, J. Arthur, R. Bionta, C. Bostedt, J. Bozek, A. Brachmann, P. Bucksbaum, R. Coffee, and F.-J. Decker, Nat. Photonics 4, 641 (2010).

15. H.-P. Geng, J.-H. Chen, and Z.-T. Zhao, Nucl. Sci. Tech. 31, 88 (2020).

16. K.-J. Kim, Phys. Rev. Lett. 57, 1871 (1986).

17. C. Feng and H.-X. Deng, Nucl. Sci. Tech. 29, 160 (2018).

18. L. H. Yu, Phys. Rev. A 44, 5178 (1991).

19. G. Stupakov, Phys. Rev. Lett. 102, 074801 (2009).

20. C. Feng, H. Deng, M. Zhang, X. Wang, S. Chen, T. Liu, K. Zhou, D. Gu, Z. Wang, and Z. Jiang, Phys. Rev. Accel. Beams 22, 050703 (2019).

21. P. R. Ribič, A. Abrami, L. Badano, M. Bossi, H.-H. Braun, N. Bruchon, F. Capotondi, D. Castronovo, M. Cautero, and P. Cinquegrana, Nat. Photonics 13, 555 (2019). 
22. E. Hemsing, A. Knyazik, M. Dunning, D. Xiang, A. Marinelli, C. Hast, and J. B. Rosenzweig, Nat. Phys. 9, 549 (2013).

23. P. R. Ribič, D. Gauthier, and G. De Ninno, Phys. Rev. Lett. 112, 203602 (2014).

24. E. Hemsing and A. Marinelli, Phys. Rev. Lett. 109, 224801 (2012).

25. Z. Zhao, D. Wang, Q. Gu, L. Yin, M. Gu, Y. Leng, and B. Liu, Appl. Sci. 7, 607 (2017).
26. J. W. Goodman, Introduction to Fourier Optics (Roberts and Company, Englewood, CO, 2005).

27. X. Wang, C. Feng, C.-Y. Tsai, L. Zeng, and Z. Zhao, Phys. Rev. Accel. Beams 22, 070701 (2019).

28. L. Zeng, C. Feng, X. Wang, K. Zhang, Z. Qi, and Z. Zhao, Photonics 7, 117 (2020).

29. S. Reiche, Nucl. Instrum. Methods Phys. Res. A 429, 243 (1999). 